\title{
Photo acknowledgements
}

The editors, contributing authors and publisher wish to express their thanks to the below sources of illustrative material and/or permission to reproduce it.

Photo Clare Archibald: p. 229; photo Jenna C. Ashton: pp. I55, 233; photo Natalie Bradbury: p. 95; photo Cassie Britland: pp. 46, I78; photo Paul Dobraszczyk: pp. 3-24, 35, 5I-4, 6I, 69-83, гоo, Io9, II7-28, I36-42, I50, I65, I86-94, 207, 222, 24I-50, 267, 276 , 284-8, 299-304, 312, 326-7; photo Nick Dunn: pp. 42, 91, 202, 259; photo Tim Edensor: pp. 86, 212; photo Steve Hanson: pp. 307; photo Richard Hopkins: p. 290; photo Joanna Hudson: pp. 210, 254; photo Peter Kalu: p. I33; photo Matthew Kowalczuk: p. 280; courtesy Manchester Metropolitan University: p. 64; courtesy Manchester Metropolitan University Special Collections: p. 294; artwork Sean R. Mills: pp. 31, I98, 237; photo Brian Rosa: pp. I46, I73; artwork Morag Rose: pp. 27, I05; photo Darmon Richter, p. I68; photo Jonathan Silver: pp. 38, 58, 317; photo Matthew Steele: pp. 160-3, 217, 271; courtesy University of Manchester: p. II2; photo Tim Woodward: p. 322. 